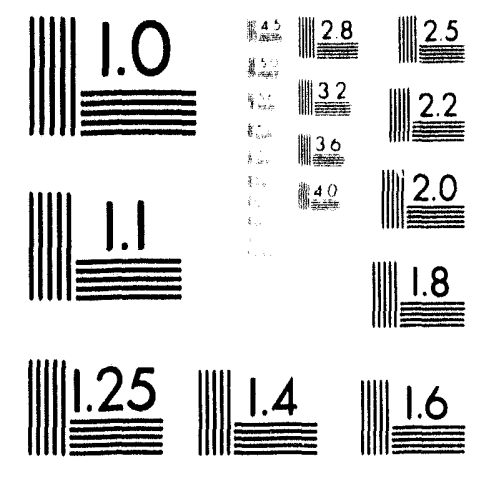




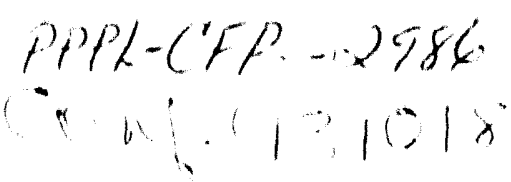

\title{
Two Frequency ICRF Heating of D-T Plasmas on TFTR*
}

\author{
J. H. Rogers, R. Majeski, J. R. Wilson, J. C. Hosea, G. Schilling, J. Stevens \\ Princeton Plasma Physics Laboratory \\ P. O. Box 451, Princeton, NJ 08543
}

\author{
Y. L. Ho \\ Science Application International Corporation \\ 10260 Campus Point Drive \\ San Diego, CA 72121 \\ S. Raman ${ }^{+}$ \\ University of Michigan \\ Ann Arbor, Michigan 48109 \\ D. A. Rasmussen \\ Oak Ridge National Laboratory \\ P. O. Box 2009, Oak Ridge, TN 37831
}

\begin{abstract}
". "difications have been made to allow two of the ICRF ante $s$ (bays $L$ and $M$ ) on TFTR to operate at either of two frequencies, $43 \mathrm{MHz}$ or $64 \mathrm{MHz}$. This was accomplished by lengthening the resonant loops feeding the antennas ( $2 \lambda$ at 43 $\mathrm{MHz}, 3 \lambda$ at $64 \mathrm{MHz}$ ) and replacing the conventional quarter wave impedance transformers with a tapered impedance design. The other two antennas (bays $\mathrm{K}$ and $\mathrm{N}$ ) will operate at a fixed frequency, $43 \mathrm{MHz}$. The two frequency operation allows a combination of ${ }^{3} \mathrm{He}$-minority (or T second harmonic) and H-minority heating at full toroidal field on TFTR. Multiple frequency operation may also be useful in direct electron heating and current drive experiments. Other modifications have been made which are expected to permit arbitrary phasing between the current straps on bays $M$ and $L$. The system design of the antenna, resonant loops and impedance matching system as well as preliminary TFTR results are discussed.
\end{abstract}

\section{INTRODUCTION}

During full powered super shots on TFTR, $64 \mathrm{MHz}$ fast waves can be used for on axis $\mathrm{H}$-minority heating and 43 $\mathrm{MHz}$ fast waves can be used for on axis ${ }^{3} \mathrm{He}$ minority heating, or in the absence of a ${ }^{3} \mathrm{He}$ puff, direct electron or second harmonic tritium heating. The ICRF heating efficiency may

\footnotetext{
* Manuscript received October 14, 1993. This work was supported by the U.S. Department of Energy under contracts N(. 1)E AC(O2. 76-CHO-3073 and DE-F(0)5-93ER 54201.

+ DOE Magnetic Fusion Technology Fellow
}

be enhanced because dividing the power between two ion species may result in the minority ions having lower energy and therefore longer confinement time and better coupling to the bulk ions. Direct electron heating and current drive experiments may also benefit from two frequency operation. Coupling ICRF power directly to the electrons is difficult unless the thermal electron energy is greater than a few keV. One frequency can be used for H-minority heating, which is effective in raising the electron temperature. Because of the strong electron temperature dependenct, the other frequency then couples power directly to the electrons more efficiently. Operating at two frequencies is also expected to reduce cross talk between the antennas. Initial operation of the system described here on TFTR has confirmed that this is a viable approach for multifrequency operation.

\section{SYSTEM DESIGN}

Each ICRF antenna on TFTR consists of a pair of current straps grounded at the center (at the midplane of the tokamak). The straps are excited by a resonant loop formed by a transmission line connected to the top and bottom of the strap (see Fig. 1). In the past, these loops were one wavelength long at the operating frequency, $47 \mathrm{MHz}$. Since two of our four antennas are fed by four tunable FMIT transmitters (one transmitter per strap, 40-80 $\mathrm{MHz}$ ), the loop configuration was changed. The resonant loops on bays $L$ and $M$ have been modified to be approximately iwice as long electrically, so that they are two and three wavelengths long for frequencies 43 and $64 \mathrm{MHz}$ respectively. During full power supershots at full toroidal magnetic liek, 4.3 MHz can provide on axis ${ }^{3}$ He-minority heating (or, with no ${ }^{3}$ lle 


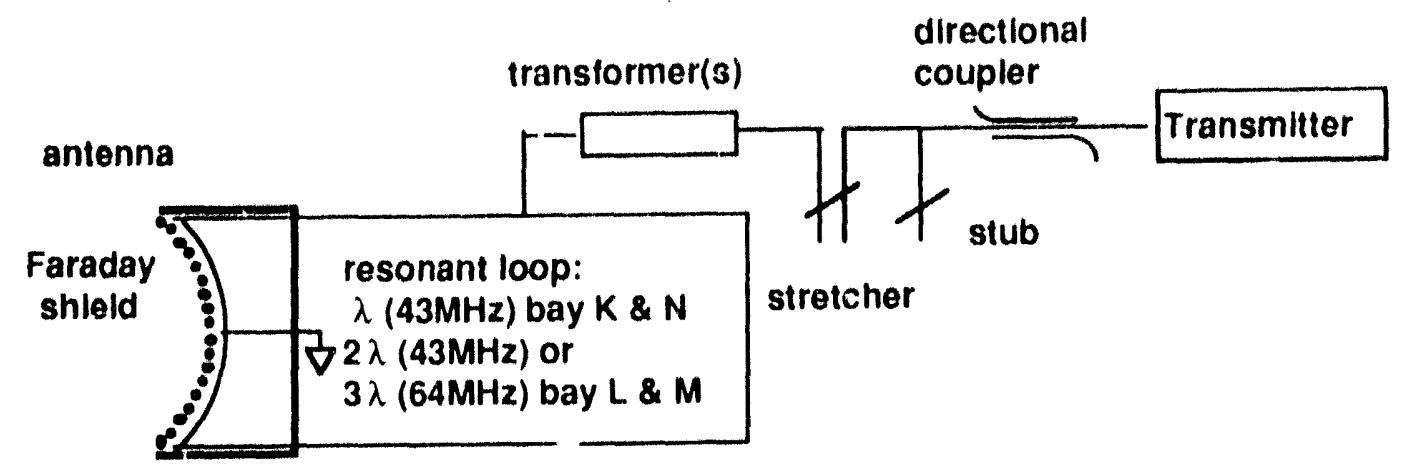

Fig. I Schematic of the TFTK KC RF antenna system

injected, tritium second harmonic healing) and $64 \mathrm{MHz}$ can provide $\mathrm{H}$-minority on axis heating.

The length of transmission line loop was chosen to be resonant at $43 \mathrm{MHz}$ ( $2 \lambda$ long elecurically). The next higher frequency harmonic of interest ( $3 \lambda$ long) is not exactly 1.5 times this frequency because the antenna straps are not well represented by a transmission line. Instead, the antenna is better approximated by a collection of lumped elements [1] and the electrical length is nonlinear. The nonlinearity of the electrical length is dependent on the details of the antenna geometry. In fact, the resonant frequency for bay $M$ is 63.38 $\mathrm{MH} \%$ and bay $\mathrm{L}$ is $63.85 \mathrm{MH} \%$.

The desired mode to be excited in the resonant loop has no net current in the midplane ground post, i.e. the top and bottom voltages are $180^{\circ}$ out of phase. In order to excite this mode, it is necessary to feed the power to the loop near a high voltage point so that the path length from the feed point to the top and bottom are $\sim \lambda / 2$ different. By symmetry, the midpoint of the transmission line in the loop is a low voltage point for both frequencies, however there is no point in the loop which is a high voltage point for both frequencies. For the antennas operating at two frequencies, the optimum feed point to the loop is $.2 \lambda$ at $43 \mathrm{MHz}(=.3 \lambda$ at $64 \mathrm{MHz})$ away from the midpoint of the transmission line, which is .05 $\lambda$ away from a high voltage point for ach fiequency. The input impedance near a high vollage peint of a resonant loop is real and large, on TFIR, typically $\sim 3 \mathrm{k} \Omega 2$ for vacuum conditioning and $\sim 25() \Omega$ for operation into a plasma. In order to reduce the VSWR in the transmission line, transformers are used near the resonant loops to bring tolai impedance down to $~ 5() \Omega$ during plasma operation $(-6()) \Omega$ during vacuum conditioning).

The bay $K$ and $N$ antennas are each led by a single transmitter. A section of transmission line $\lambda / 2$ long connects the two feed points of the two resonatul loops for each antenta (fixing the phase hetween straps al ()-18(1) $)$. Because the two resonant loops are fed in parallel, the expected impedance is

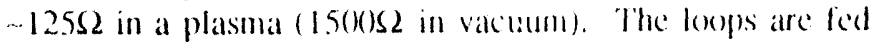

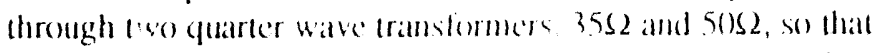
the $5($ s) line is matched when the leed point on the loop has an impedance of 102.82.

bach strap on hay 1 and $M$ ane led hy a separalle transmiller. Broad hand tramslommers are med lo leced the

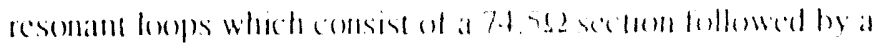

149.1 2 section, each of which is a quarter of a wave length long at 53.5 MHz. This arrangement is optumized for a impedance at the loxp input of 24 ()S2 at either $43 \mathrm{MHz}$ or 64 MHz. The details of this transformer design as well as a lumped element model of the antennas has been presented elsewhere [1].

Each transmitter has a stub and stretcher for fine tuning the impedance match. A directional coupler on the ransmitter side of the stub is used to measure the forward and reflected voltage and the phase difference between them. These values determine a load impedance, and the appropriate stub and stretcher positions are calculated to match this impedance. However, there is a complication on the antennas in which a separate transmitter feeds each side. The resonant behavior of the loops is a strong function of the relative phase between the straps, so small changes in the phase can change the input impedance. By symmetry, during $0-()$ or $0-180^{0}$ phase operation, the correct match should be obtained with both sides having the same stub and strecther positions. Thus, if both sides start with the same stub and stretcher positions, and equal corrections are made on both sides, the correct match can be calculated assuming a constant load.

ICRF last wave current drive is an important component of future tokamaks (ITER and TPX). The coupling to the electrons is strongly affected by the component of the wave vector in the toroidal direction, $k_{\|}$. The $k_{\|}$spectrum, including directivity, can be modilied by varying the phase between the two current straps in the antenna. However, because of the mutual coupling between the straps, the resonant behavior of the loops, and the VSWR between the stub and the loops, there is not a simple correspondence between the phase difference of the transmitters and the phase difference of the straps. In fact, changing the matching clements o improve the impedance match will result in changing the strap phase, which effectis the mulual coupling, which effects the apparent impredance of the amenna, making it very difficult to find the apprepriale impedance match. In

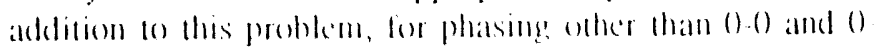

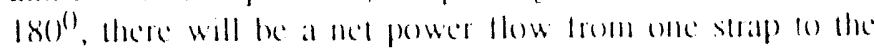

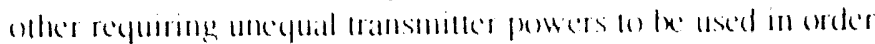

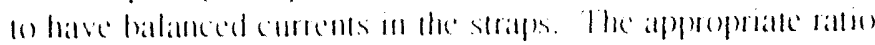

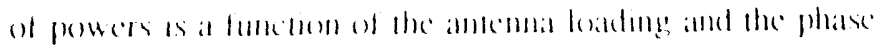

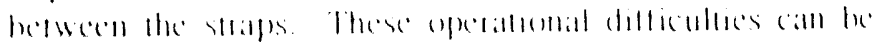



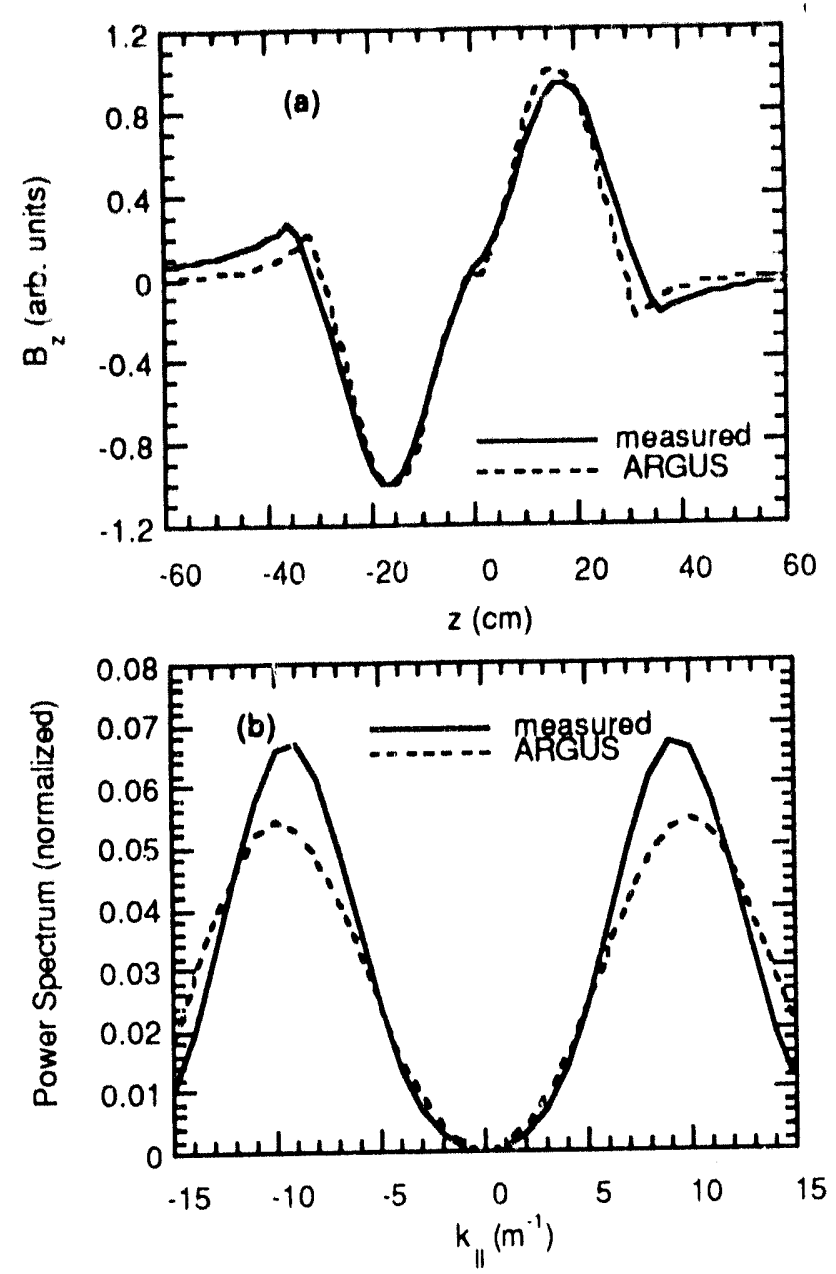

Fig. $2 \mathrm{~B}_{\mathrm{z}}$ for $0-180$ phasing measured $1 \mathrm{~cm}$ from the faraday shield at the midplane of a mock up of the bay $M$ antenna and the field calculated by the ARGUS code (a), Also, the fourier transform of the square of the fields (b).

minimized by phase locking the transmitters with reference to the phase between the antenna straps and using amplitude leveling based on the antenna voltage. By forcing the phase between the straps to be constant, and the amplitudes constant, the coupling between the straps is forced to remain constant. The impedance of each resonant loop must then stay constant from shot to shot, and the correct stub and stretcher positions can be calculated using the measured reflection coefficient in the usual way [2].

\section{ANTENNA VACUUM FIELDS}

In order to model the direct electron heating and current drive, it is impor'ant to know the $k_{\|}$spectrum of the launched wave. The B-fields were carefully measured $1 \mathrm{~cm}$ from the faraday shield of a mock-up of the bay $M$ antenna. Calculations were also performed to estimate the fields in front of the antenna using the ARGUS modeling code |3|. Fig. 2 shows the measured and calculated $B$, field at the antenna midplane with $\left(0.180^{(0)}\right.$ phasing hetween the straps. Fig. 2 also shows the fourier transform of this lield in the ,
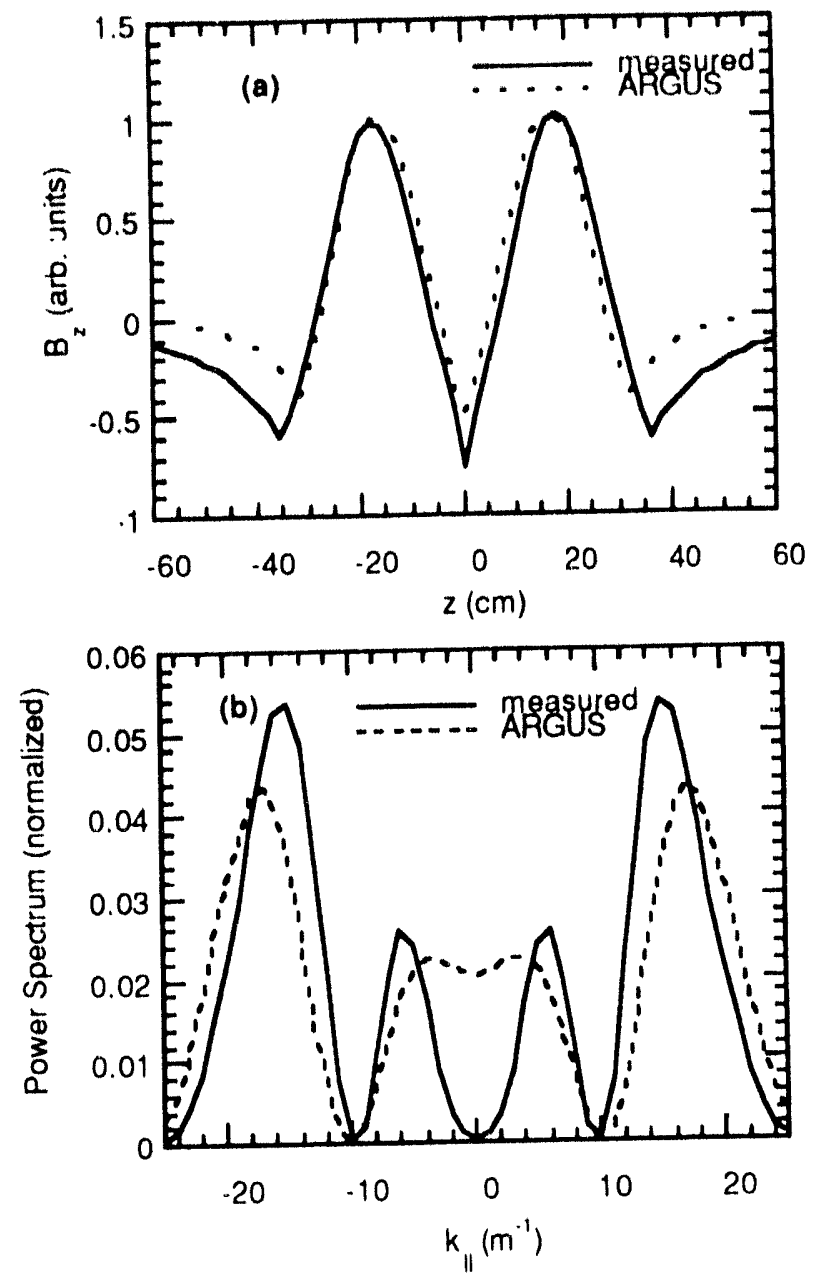

Fig. $3 \mathrm{~B}_{\mathrm{z}}$ for $0-0$ phasing measured $1 \mathrm{~cm}$ from the faraday shield at the midplane of a mock up of the bay $M$ antenna and the field calculated by the ARGUS code (a). Also the fourier transform of the square of the fields (b).

(toroidal) direction squared (giving the power spectrum). It can be seen that the $\mathrm{B}_{Z}$ field appears to have approximately a sinusoidal dependence with half a wavelength equal to the center to center strap separation $(0.33 \mathrm{~m})$. This is verified by the peak of the $\mathrm{k}_{\|}$spectrum at $\sim 9.5 \mathrm{~m}^{-1}$. Fig. 3 shows the $B_{\text {, }}$ field at the midplane of the antenna with $\left(0-0^{(0}\right.$ phasing between the straps, and the corresponding $k_{\|}$spectrum. Even though the septum between the straps is slotted perpendicular to the direction of the current to a depth $5.4 \mathrm{~cm}$ behind the straps, the image currents in the septum still perturb the fields significantly. Whereas it may have been expecied that the dominant wave length for $\left(0-()^{0}\right.$ phasing would have been longer than for $0.180^{()}$phasing, in fact the majority of the power is associated with shorter wavelength (larger $k_{\|}$). Also, even though the side walls and septum between the straps are sloted, the image currents generatle enough ficlds out of phase with the strap fields that the spectral power gees to gero for $k_{i l}$ $=0$. The ARCiUS calculations retain a finite power for $k_{\|}=0$ ). This difference in $k_{1 i}$ may be dee wo the bendary conditions in ARcius which include a vacumul vessed wall, while the measurements were makte on a the tanding antentat 

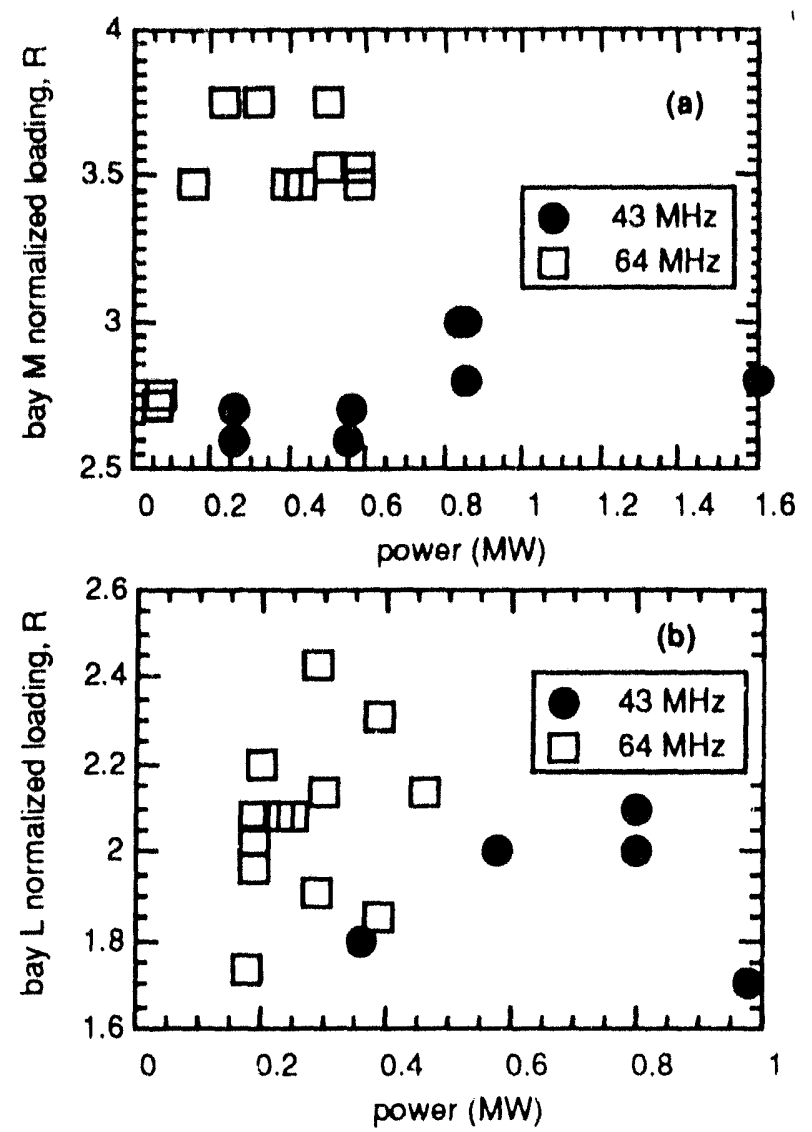

Fig. 4 The measured loading of the bay $M$ (a) and bay $L$ (b) antennas relative to the minimum necessary to achieve maximum power versus the power transmitted out of that bay.

\section{INITIAL TFTR RESULTS}

Bays $M$ and $L$ have now been operated at both 43 and 64 $\mathrm{MHz}$ with $0-180^{\circ}$ phasing. Measurements of the voltage and current at the top and bottom of each strap indicate good balance of the currents on the straps for each frequency.

In order to quantify the loading in some meaningful way, it can be noted that the antenna loading is proportional to $P / V^{2}$, where $P$ is the transmitted power and $V$ is the maximum voltage in the resonant loop. This value can be normalized by the smallest acceptable loading defined by the antenna reaching the maximum rated voltage at the maximum transmitter power. On TFTR, $V_{\text {max }}=50 \mathrm{kV}$ and $P_{\max }$ on bays $\mathrm{M}$ and $\mathrm{L}$ is $3.5 \mathrm{MW}$. Then as long as $\mathrm{R}$ is greater than one, maximum transmitter power should be achievable, where $\mathrm{R}=\left(\mathrm{P} / \mathrm{V}^{2}\right)\left(\mathrm{V}_{\max } 2 / \mathrm{P}_{\max }\right)$. This normalized loading is shown in Fig. 4 for both antennas and both frequencies during $\mathrm{H}$ minority heating (the higher frequency requires a higher toroidal field to maintain on axis heating). It can he seen that the loading at both frequencies is comparable for the plasma conditions chosen for the two cases. Bay $M$ has somewhat higher loading than bay $L$ because the strap separation is somewhat larger $[4]$. Because $R>1$, the antenna loading al each frequency is sufficient to allow the antennas to opprate at full available power and remain below their maximum rated voltage.

\section{REFERENCES}

[1] J. H. Rogers, R. Majeski, J. R. Wilson, J. C. Hosea, G. Schilling, J. Stevens, and D. A. Rasmussen, "Two frequency ICRF operation on TFTR," Proc. of the $10^{\text {th }}$ Topical Conf. on RF Power in Plasmas, Boston MA.1.3 April 1993, in press.

[2] D. M. Pozar, Microwave Engineering, Reading: AddisonWesley, 1990, PP. 288-292.

[3] A. Mankofsky, et. al, in Linear Accelerator and Beam optics Codess, ed. C. R. Eminheizer, A. I. P. Conference Proceedings No. 177 (A.I.P., New York, 1988), pg. $137 \mathrm{ff}$. And C. L. Chang, et. al., in Proceedings of the Conference on Computer Codes and the Linear Accelerator Community, ed. R. K Cooper (Los Alamos National Laboratory report, LA-11857-C', January 22. 25, 1990), pg 27.

[4] J. H. Rogers, J. R. Wilson, J. Stevens, J. C. Hosea, G. Schilling, C. K. Philips, D. J. Hoffman, and D. Rasmussen, Proc. of the Europhysics Topical Conf. on RF Heating and Current Drive, Brussels, Belgium 7-10 July, 1992.

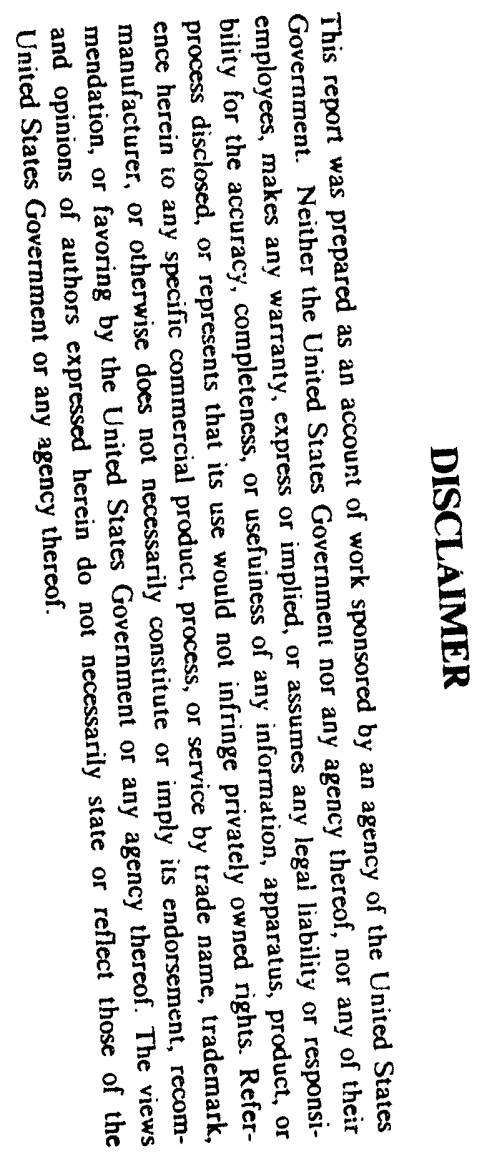



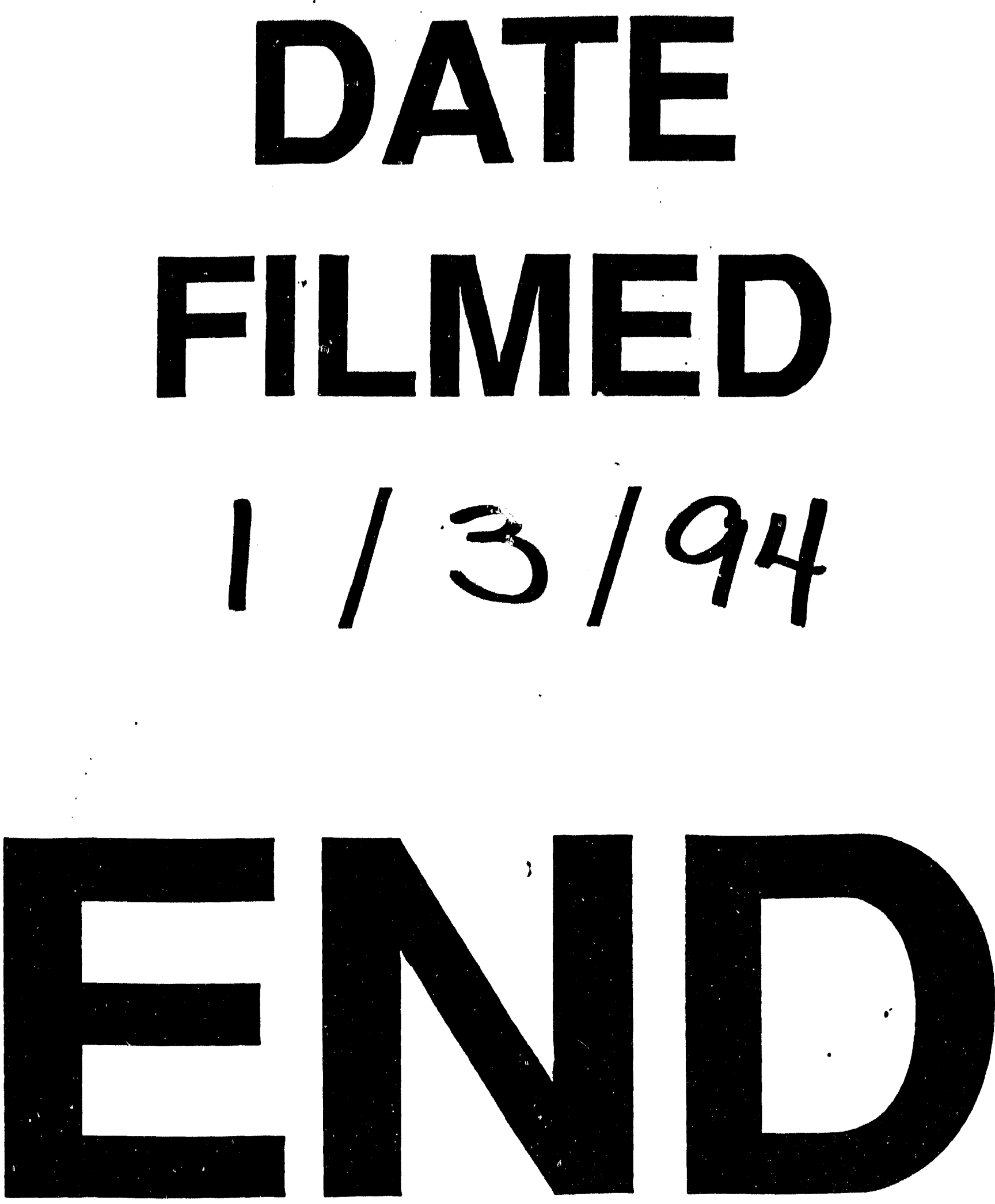


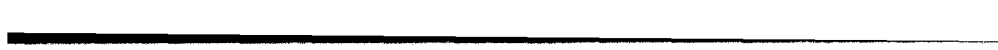

1 